\title{
Motor shaft vibrations may have a negative effect on ability to implement a stiff haptic wall
}

Book or Report Section

Accepted Version

Swaidani, L., Steele, L. and Harwin, W. (2018) Motor shaft vibrations may have a negative effect on ability to implement a stiff haptic wall. In: Haptics: Science, Technology, and Applications. Lecture Notes in Computer Science, 10894. Springer, pp. 252-263. ISBN 9783319933986 doi: https://doi.org/10.1007/978-3-319-93399-3_23 Available at http://centaur.reading.ac.uk/85970/

It is advisable to refer to the publisher's version if you intend to cite from the work. See Guidance on citing.

To link to this article DOI: http://dx.doi.org/10.1007/978-3-319-93399-3_23

Publisher: Springer

All outputs in CentAUR are protected by Intellectual Property Rights law, including copyright law. Copyright and IPR is retained by the creators or other 
copyright holders. Terms and conditions for use of this material are defined in the End User Agreement.

\section{www.reading.ac.uk/centaur}

\section{CentAUR}

Central Archive at the University of Reading

Reading's research outputs online 


\title{
Motor shaft vibrations may have a negative effect on ability to implement a stiff haptic wall
}

\author{
Louis Swaidani $^{1}$, Luke Steele ${ }^{2}$, and William Harwin ${ }^{1}$ \\ 1 Department of Biological Sciences, University of Reading, Reading, United \\ Kingdom, \\ louis.swaidani@pgr.reading.ac.uk \\ ${ }^{2}$ Generic Robotics, Building L014, University of Reading, London Road Campus, \\ Reading RG1 5AQ, United Kingdom, \\ L.Steele@genericrobotics.com
}

\begin{abstract}
A one degree of freedom experimental test bed is used to investigate the effects of elastic vibration in haptic devices. Strong angular vibration occurs at the motor rotor due to elastic deformation in the shaft. These vibrations occur due to large discontinuities in the virtual environment such as stiff contact which is common in haptics. Also looked at was the effect of these vibrations on stability and control. It was found that the vibrations may negatively affect the stability of the haptic device by introducing large measurement errors to the controller. The experiments investigated using different types of damping in controller feedback. Adding damping to the system whilst these elastic vibrations are present can successfully damp the system, but also tend to increase the magnitude of vibrations sometimes resulting in greater instability. Finally, a second non co-located encoder was used to try to eliminate measurement error from the system due to vibration. It was found that by simply placing the encoder closer to the link where the angle is being measured, error due to rotational flex in the shaft is eliminated. This yielded the greatest improvement in controller performance, nearly eliminating the presence of the vibrations and their effects.
\end{abstract}

Keywords: Stiffness · elastic · torsional deflection · vibration · limit cycle $\cdot$ gyro $\cdot$ damping $\cdot$ non-collocated control $\cdot$ haptic device control . modelling

\section{Introduction}

Haptic interfaces provide a tactile and proprioceptive mode of interaction with a virtual environment. One of the persistent challenges with haptics is producing consistently realistic contact with objects in the virtual environment whilst eliminating instability and unwanted vibration.

There has been much research on the stability of haptic devices with regards to the controller. Time delay, quantisation error and interaction with the human operator have all been cited as causes of instability or causes of non-passive behaviour $[1-6]$. There is less in the literature looking at the effects of stiffness 
and elastic deformation of the haptic device itself potentially resulting in vibrations. These vibrations can affect the kinematics of the device by introducing measurement error into the control system or by storing energy elastically and releasing it later. This can ultimately impact on stability of the system. In addition to this, vibration is important to the user's haptic perception of the virtual environment.

Elastic vibration can occur in systems where inertial bodies are connected by stiff components. In haptic devices this is usually in the transmission, such as a shaft or cable drive rather than the links themselves. Whilst elastic deflection of the linkages are known to contribute to error in end effector position and poor trajectory tracking in robotics[7] in addition to adding vibrations, the case of flexible joints and transmissions is of more relevance to the majority of haptic applications.

Haptic interfaces, like the W5D from Entact Robotics, have the links of the device directly mounted on the motor shaft whereas devices like the PHANToM are driven by steel cable drives. The elastic strains resulting from normal operating conditions have a significant effect on kinematics and control of the device as well as setting up elastic vibration. Angular vibrations can the occur both in the motor rotor or the links of the device. Cable drives are noticeably elastic in nature and thus haptic or tele-robotic systems designed in this way exhibit significant error in end effector position. Shaft driven transmissions do not suffer significant error in end effector position due to having relatively high stiffness but the small deflections that do occur can lead to instability in the haptic controller producing strong vibrations that can be seen and felt by the user.

One of the difficulties with haptics is implementing stiff contact with virtual objects whilst avoiding unstable behaviour[8]. It is possible that vibration in the haptic device plays some role in this instability. Stiff contact introduces a sudden change in torque to the haptic device. Similar shock-loading usually leads to harmonic vibrations in non-rigid systems, this may be responsible for some of the vibrations found in haptic devices.

The purpose of this paper is to investigate the cause of these vibrations, and evaluate their effects on the stability of a haptic device. Furthermore, it intends to develop solutions to minimise the adverse effects of these vibrations by investigating different types of sensor for damping and the advantages of non-collocated sensors and actuators.

\section{Methods}

\subsection{Haptic interface design}

The experimental testbed used was a one degree of freedom (1-DOF) device consisting of a single motor attached to a lever arm which the user would normally operate holding at the tip shown in Figure 1. This is similar to a 'paddle' style haptic device [9] and, due to its simplicity, is good for looking in detail 


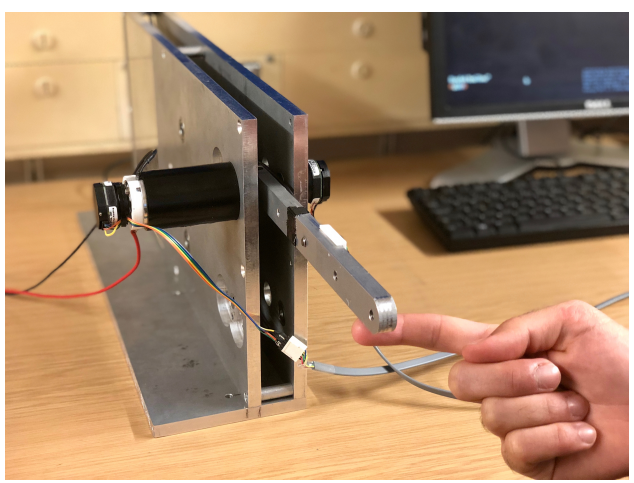

Fig. 1. The experimental test bed which is a 1-DOF haptic interface. The user holds the tip of the beam and moves it till reaching the haptic wall where the motor is turned on.

at vibrations in the device and subtle changes in sensors or the controller. The construction of the device is robust to eliminate the effects of vibrations or play anywhere apart from the unconstrained motion of the motor (Maxon RE40 148877) and end effector. The beam used was also designed to have a high enough stiffness to eliminate any vibration in the beam. The beam was $200 \mathrm{~mm}$ long with a cross section of $10 \mathrm{~mm}$ by $20 \mathrm{~mm}$ giving it a second moment of inertia of $1.66 \times 10^{-3} \mathrm{~mm}^{4}$. The aluminium beam is mounted directly to the motor shaft and the motor shaft itself is doubly supported with ball bearings; as shown in Figure 1. Figure 2 shows a free body diagram of the moving components in the

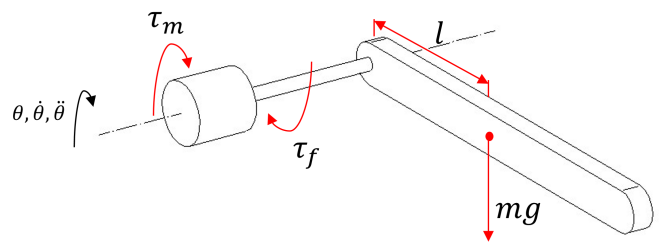

Fig. 2. Free body diagram of the moving components of the haptic device. Depicted are the motor rotor, motor shaft and the solid beam which constitutes the end effector. $\tau_{m}$ is the motor torque and $\tau_{f}$ is the torque applied at the other end of the motor shaft.

device. Assuming the shaft as a rigid body (ignoring stiffness) the motor rotor, shaft and beam can be modelled as a single body. However, considering stiffness, the device can be considered as two inertial masses connected by a stiff shaft as shown in Figure 3. In this system, the inertial bodies may rotate separately with the first (the motor) as $\theta_{1}$ and the second (the beam) as $\theta_{2}$. The rotational movements of the two are constrained only by the stiffness and damping terms 
related to the shaft. The stiffness of the $6 \mathrm{~mm}$ diameter shaft was calculated as $143.59 \mathrm{Nm} / \mathrm{rad}$.

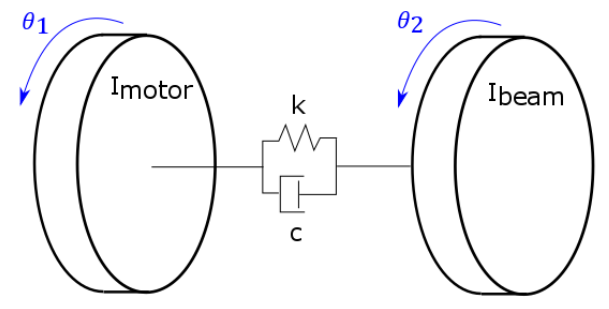

Fig. 3. Simplified model of the haptic device as a two mass system connected by a stiff shaft (represented here with a spring and dashpot). Where $I_{\text {motor }}$ and $I_{\text {beam }}$ are the respective inertial masses of the motor rotor and the beam, $k$ is the angular stiffness of the motor shaft and $c$ is an angular damping term.

\subsection{Sensors and placement}

Encoders are commonly used, in haptics and other control applications, for accurate measurement of the position of the motor. They are usually mounted on the motor itself; an arrangement provides a precise measurement of the position of the motor but in a system that is sufficiently non-rigid may lose accuracy if trying to measure end effector position. The haptic device was fitted with two identical encoders for these experiments, one at normal mounting location at the rear of the motor (Encoder 1) and one at the distal end of the shaft (Encoder 2).

Both of the encoders used were identical 20000count/rev quadrature encoders (Quantum Devices LP12-20000-A-C-D-C-A). An additional gyro sensor was fitted on the beam about halfway along the length. The gyro sensor used was Analog Device's ADXRS613. The range of which is $\pm 150 \mathrm{deg} / s$ with a sensitivity of $12.5 \mathrm{mV} / \mathrm{deg} / \mathrm{s}$ and a bandwidth of $3000 \mathrm{~Hz}$.

Adding damping to the haptic device requires a measurement of angular velocity. This is usually done by taking the derivative of the position measurement. Since this is a sampled system this is a discrete derivative and requires a small amount of low-pass filtering. The resulting velocity estimate therefore has a minimum delay of half the sample time but with the filtering is about $3.5 \mathrm{~ms}$ when running the control loop at $1000 \mathrm{~Hz}$. The resolution of the encoder for angular velocity is $0.314 \mathrm{rad} / \mathrm{s}$. The rate measurement from the gyro did not have additional filtering and while its effective resolution depends on the noise in the analogue signal it was a factor of ten better than that of the encoder at about $0.0382 \mathrm{rad} / \mathrm{s}$. 


\section{$2.3 \quad$ Experiments}

A set of experiments were conducted with different gains set for the stiffness and damping of the haptic device. For each experiment the end effector was placed carefully at the surface of the virtual wall (0radians) and not held by a human user. The controller was set with a high wall stiffness (high proportional gain $k$ ) which will initiate a limit cycle which will grow and settle at a marginally stable state. In this way the end effector is seen to bounce on the surface of the virtual wall and exhibit other vibrations.

The experiments were repeated under different conditions, namely which sensor was used to measure the angular position and velocity of the haptic device and feedback to the motor as well as their placement in the case of the encoders. The two encoders used, one located on the back of the motor as is commonly seen in control applications and one located at the other end of the motor shaft closest to the beam, are hereon referred to as encoder 1 and 2 respectively and their measurements as $q_{1}$ and $q_{2}$. The entire system was controlled using Matlab and Simulink with the real-time toolbox operating via a Simulink Real-time target PC fitted with a Quanser Q8 data card. The values for controller gains were chosen to produce a usable stiffness for a 'haptic wall' and so that the limit cycles of the device would settle to a marginally stable state and within reasonable time so that they can be compared.

Table 1. List of the sensors and signals used in each experiment for control of the haptic device.

\begin{tabular}{|c|c|c|c|c|c|c|}
\hline Experiment & \multicolumn{2}{|c|}{ Angle $(q)$} & \multicolumn{2}{|c|}{ Angular velocity $(\dot{q})$} & \multicolumn{2}{|c|}{ Control gains } \\
\hline & Encoder 1 & Encoder 2 & Difference filter & Gyro rate & $k_{p}$ & $k_{d}$ \\
1 & $\mathrm{x}$ & - & - & - & 800 & - \\
2 & $\mathrm{x}$ & - & $\mathrm{x}$ & - & 800 & 1 \\
3 & $\mathrm{x}$ & - & - & $\mathrm{x}$ & 800 & 1 \\
4 & - & $\mathrm{x}$ & - & - & 800 & - \\
5 & - & $\mathrm{x}$ & $\mathrm{x}$ & - & 800 & 1 \\
6 & - & $\mathrm{x}$ & - & $\mathrm{x}$ & 800 & 1 \\
\hline
\end{tabular}

\section{Results}

Figure 4 shows the a portion of the data from Experiment 1 where the end effector of the haptic device was left to rest on the virtual wall (at 0 radians) but due to the high stiffness gain $k$ and no damping (except the inherent damping of the hardware due to friction) resulted in a continuous and predictable limit cycle. This snapshot of the data is one sample of one of these limit cycles or 'bounces' on the virtual wall. The figure shows four graphs from produced from the data over the same time window, the first two of which show the states 
of the system as recorded by the sensors. Encoders 1 and 2 measured angular position in quadrature mode at 20000 counts/rev. Plot (a) compares position from encoder 1 and encoder 2 in Experiment 1, which used the ordinary motor encoder (encoder 1) for position control which is the most common mounting position. Although the signal from encoder 2 was not used for control purposes in experiment 1 it shows the actual position of the beam and end effector. The second plot (b) shows the velocity signals from the gyro and the differentiated encoder position measured by encoders 1 and 2. Plot (c) shows the deflection in the rotor shaft by taking the difference in angle measured between the two encoders. Below this plot (d) shows the feedback to the motor from the controller. The effect of adding different methods of damping and only the motor encoder for position was tested in experiments 1,2 and 3 . The angular position measurement from these tests can be seen in Figure 5 which compares the position and velocity signals of these experiments. The rest of the results displayed in Figrues 6, 7 and 8 show selected comparisons of the measured shaft deflections under different control conditions and the control signal to the motor during interaction with the haptic wall. Figure 6 compares the elastic vibrations using encoder 1 with and without damping as performed in experiments 1 and 2. Figure 7 shows the resulting reduction in vibration from switching from encoder 1 to encoder 2 without adding damping which corresponds with experiments 1 and 4 . Figure 8 compares the damping achieved using the gyro rate measurement versus differentiated encoder signal whilst using only the beam encoder (experiments 5 and 6). In each figure the upper plot (a) shows the angular deflection of the shaft whilst the end effector is resting on the whilst below (b) is the control signal $u$ to the motor.

\section{Discussion}

The results from Experiment 1 suggests that the two mass model of the test bed device can be applied to this scenario. As the encoder reading crosses zero and the controller recognises the beam as having entered the haptic wall, the motor is turned on. This, due to the high wall stiffness, results in shock loading and a large vibration in the haptic device. These vibrations are audible but can also be felt if the user holds the end effector. Figure 4 also shows that the same transient vibrations occur when the position measurement crosses zero in the opposite direction thus leaving the haptic wall and turning the motor off. This is due to the motor shaft being suddenly released from torsion. Finally, it can be seen that regardless of the scenario these transient vibrations occur at the same natural frequency as it is independent of the control parameters.

This behaviour supports the model of the haptic device as a shaft with rotation masses exhibiting harmonic vibration. The shock loading and unloading of the motor shaft due to the unilateral constraint at the haptic wall initiate the vibrations at the natural frequency of the system. The ratio of amplitude of an- 


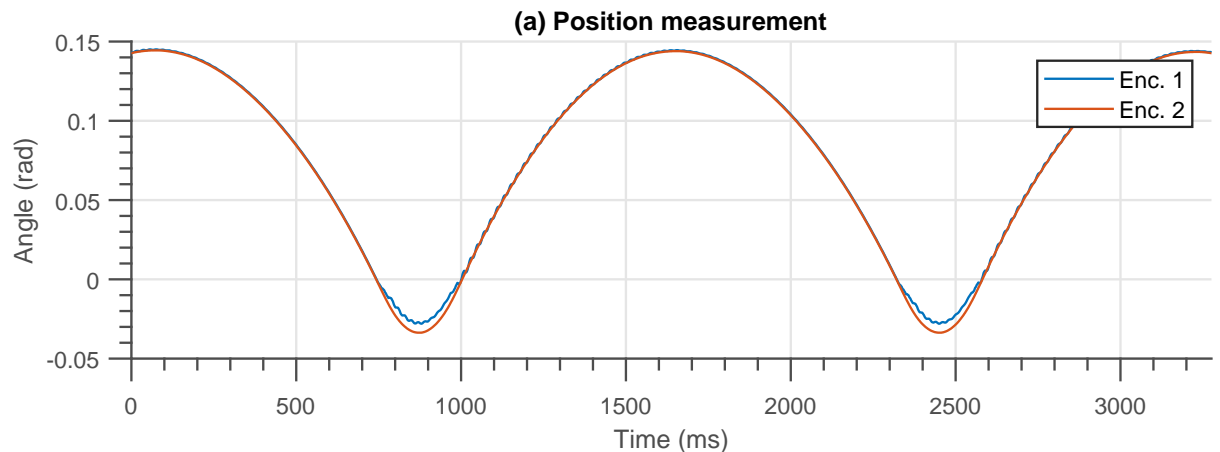

(b) Velocity measurement
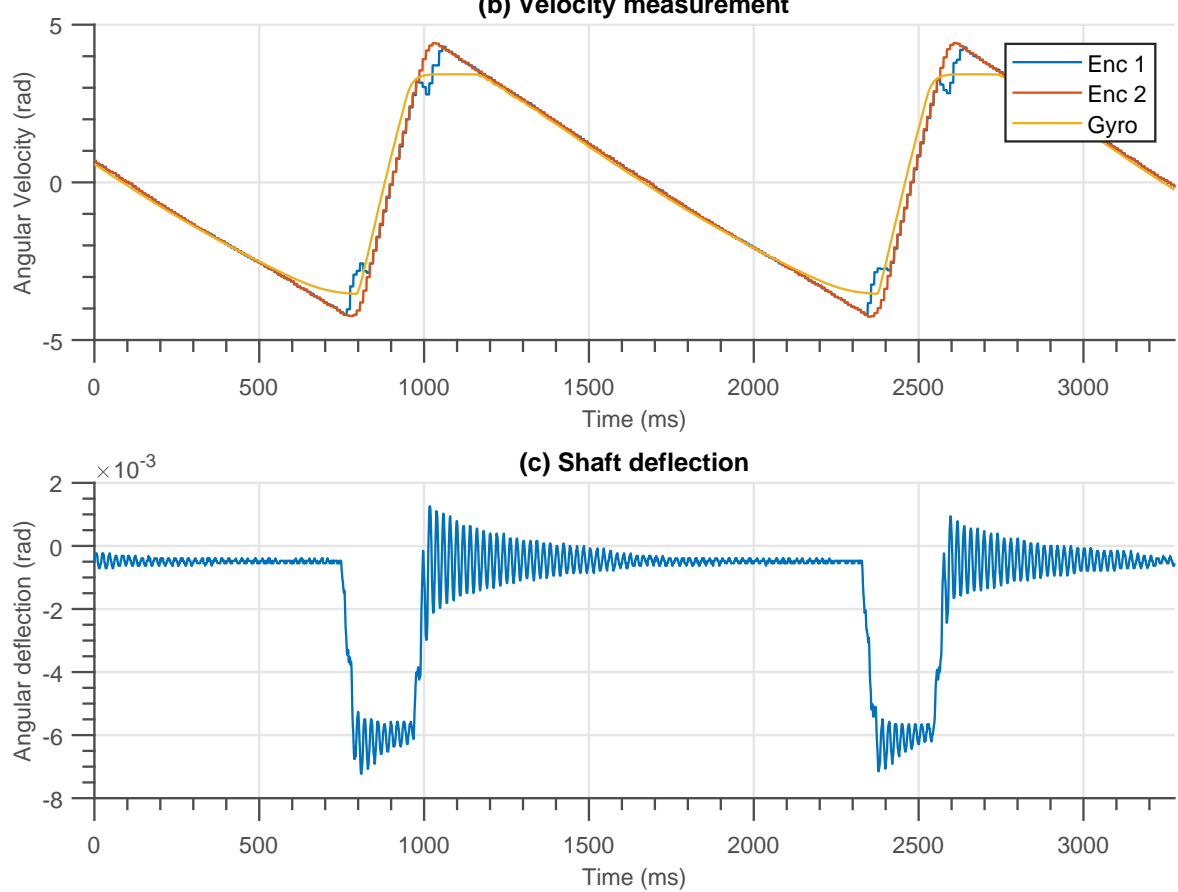

(d) Control signal

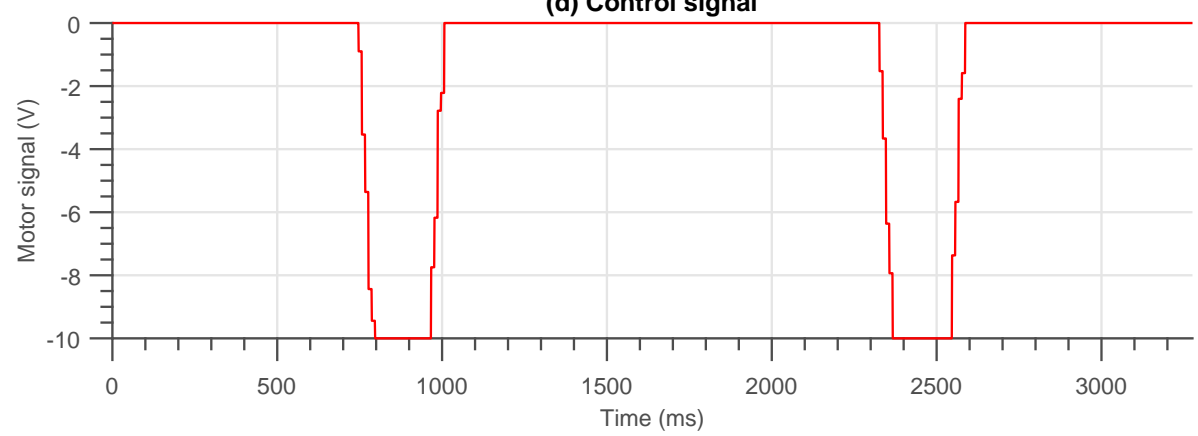

Fig. 4. Four different plots of data from experiment 1 (see Table 1): (a) plots encoder angle measurement against time (b) velocity against time from encoder differentiation and gyro sensor (c) angular deformation of the motor shaft due to torsion (d) control signal $u$ sent to the motor corresponds to angular deformation 


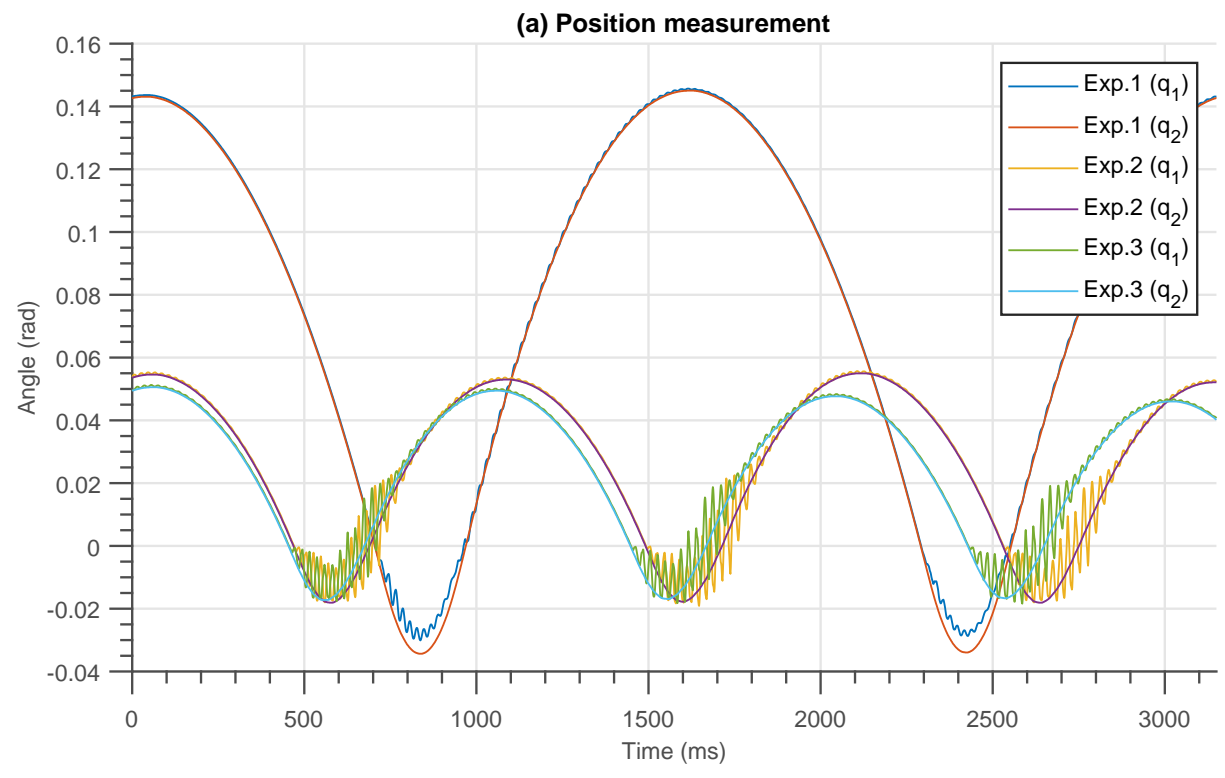

Fig. 5. Shows the angle measured for experiments 1,2 and 3 (see Table 1 ) with both encoders against time. Motor encoder is $q_{1}$ and beam encoder is $q_{2}$. Large vibrations can be seen in the motor encoder which is the motor rotor vibrating due to shock loading of the motor shaft.

(a) Shaft tortional deflection

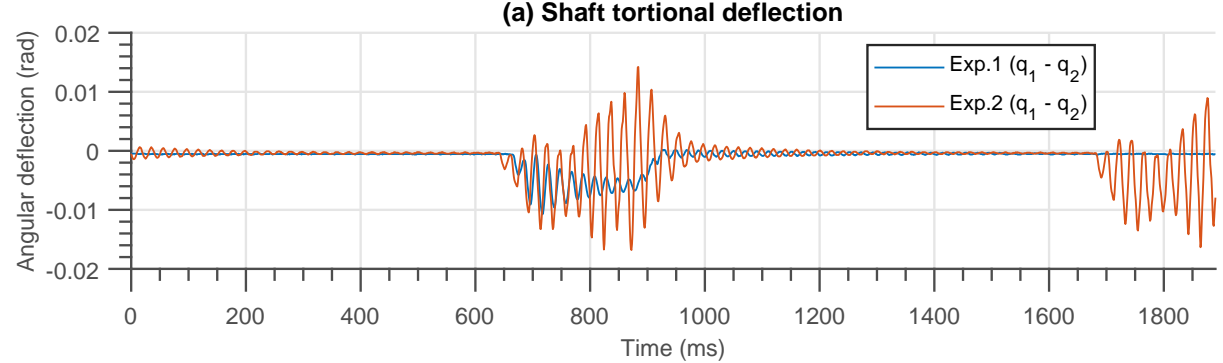

(b) Control signal

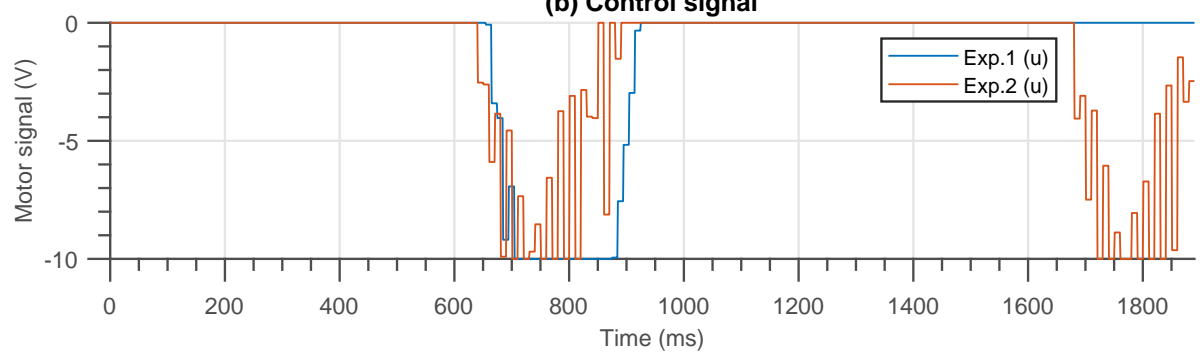

Fig. 6. The effect on vibrations and the control signal to the haptic device when adding damping and measuring position and velocity with the motor encoder. Upper plot (a) shows the angular deflection in the motor shaft. The lower plot (b) shows the control signal $u$ to the motor driver produced by the controller. 

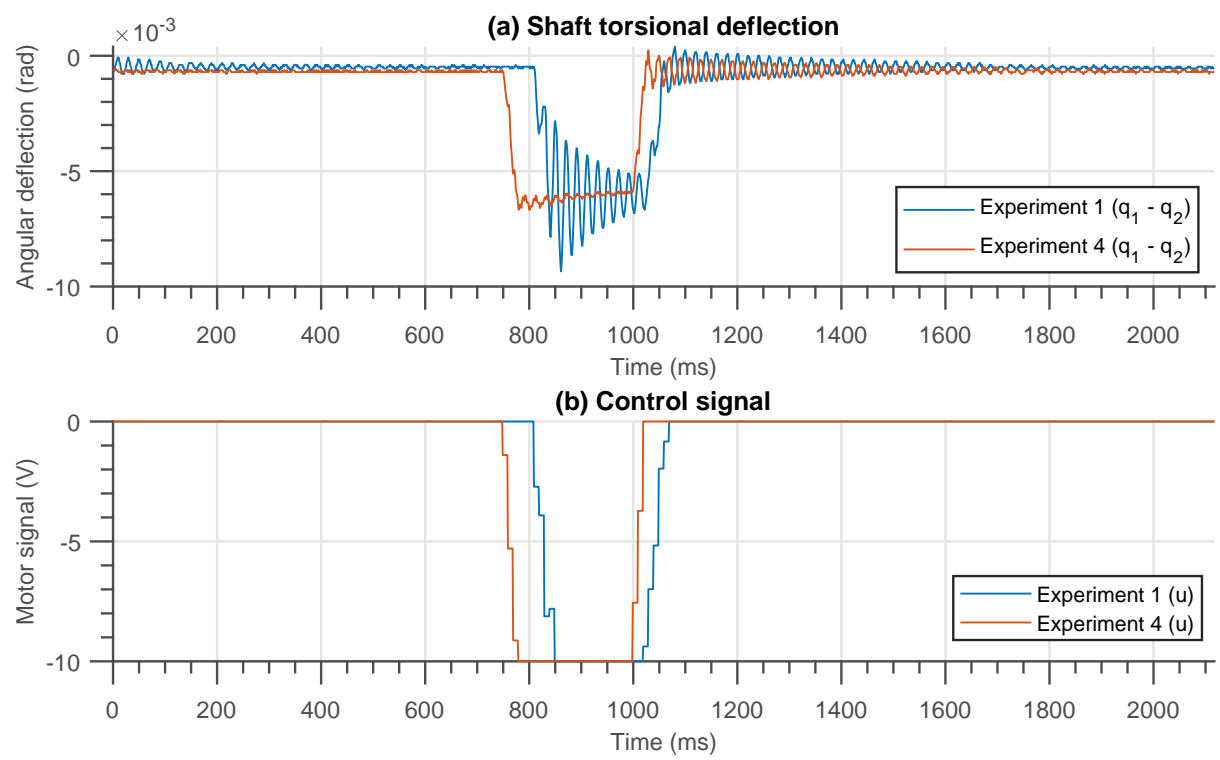

Fig. 7. Comparison of angular deformation and control signal whilst using encoders 1 and 2 for measuring angle and no damping (experiments 1 and 4). Upper plot (a) shows the torsional deflection in the motor shaft found by subtracting the difference in encoder position. Lower plot (b) shows the signal to the motor of the haptic device.
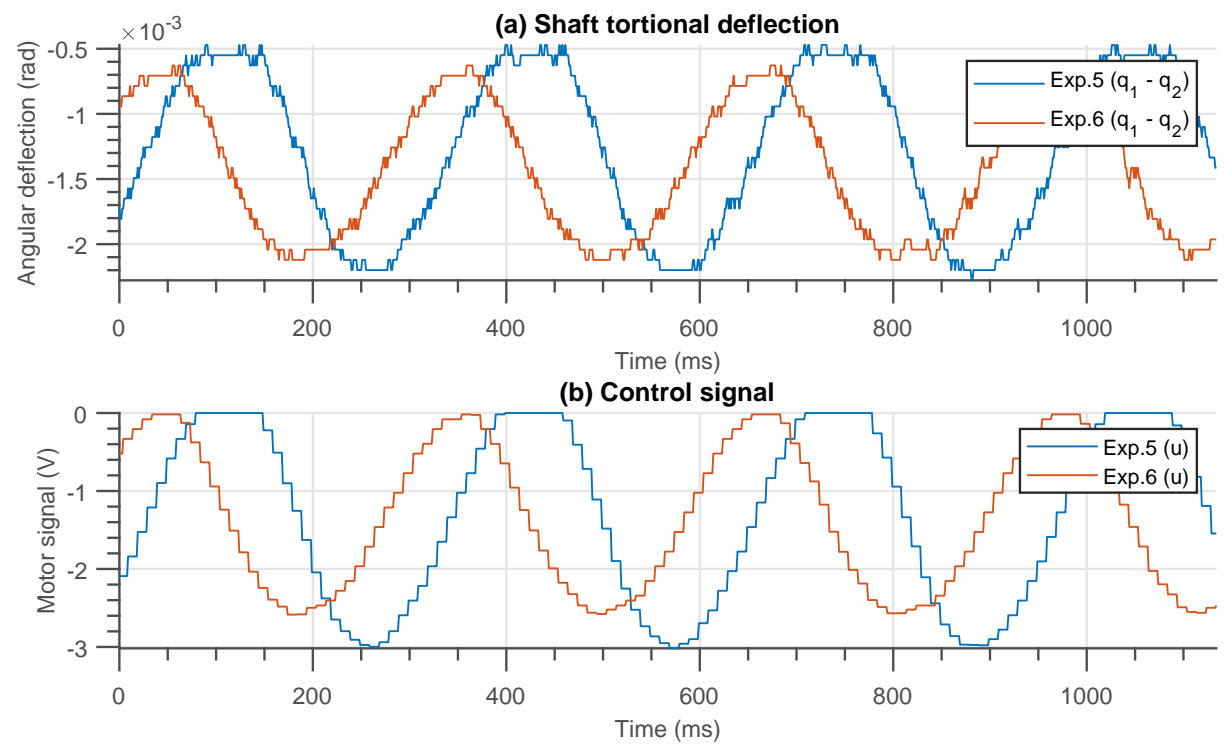

Fig. 8. Comparison of angular deformation and control signal using encoder and gyro damping with angular measurements taken from the beam encoder (experiments 5 and 6). (a) The torsional deflection in the motor shaft found by subtracting the difference in encoder position. (b) The signal to the motor driving the haptic device. 
gular vibration of the two bodies in the two mass system is given by Equation 4 .

$$
\frac{\hat{\theta}_{1}}{\hat{\theta}_{2}}=\frac{I_{2}}{I_{1}}
$$

where:

$I_{n}=$ Inertia of mass $n$

$\hat{\theta}_{n}=$ amplitude of vibration in mass $n$

The difference in inertia of the motor rotor and the beam is sufficiently large that the amplitude in the beam is $0.86 \%$ of what is seen in the motor. Thus in this particular scenario it is reasonable to simplify the model of torsional vibration from a shaft with two rotating masses to a shaft with one rotating mass and one fixed end. The base natural frequency of the system should be given by the Equation 4 .

$$
\omega_{n f}=\sqrt{\frac{k_{t}}{I_{m}}}
$$

where:

$k_{t}=$ the torsional stiffness

$I_{m}=$ the motor inertia.

Modelling the vibrations of the device using the simplified case of a shaft with one fixed end and a single rotating mass at the other gives a natural frequency equal to $503 \mathrm{~Hz}$. The data from the experiments show that the vibrations do indeed occur at approximately $500 \mathrm{~Hz}$. Crucially these vibrations are within the range that will not be filtered out by a $1 \mathrm{kHz}$ control loop and will therefore introduce oscillation in the control signal $u$ that is fed back to the motor. To ensure that the observed vibrations were not subject to aliasing as the sample rate is just high enough to observe $500 \mathrm{kHz}$ vibration, the measurement data seen here was sampled at $10 \mathrm{kHz}, 10$ times the control loop sampling rate. This did not reveal any higher frequency vibration but simply confirmed the observations.

Care was taken to ensure these vibrations were not as a result of play in the joints or loose mounting of the beam. These would also present as discontinuities in the angular motion rather than continuous elastic vibration. Looking at Figure 7 , the only discontinuity we see is when crossing the boundary at the haptic wall and after this we see simple harmonic motion which decays with time.

Adding damping to the system when using the motor encoder did not improve the suppression of the vibrations. Figure 6 shows the difference in vibrations and controller feedback that resulted from implementing damping with an encoder based velocity estimate. Whilst it did reduce the amplitude of limit cycles of the device it did not damp them sufficiently for the beam to come to rest on the haptic wall. Additionally, the amplitude of the vibrations in the motor increased. This is also seen in the lower plot (b) where the motor vibrations are so large that they introduce fluctuations in the control signal $u$. Figure 5 shows the position 
measurement for experiments 1 and 2 where we can see from the beam encoder that during each bounce or limit cycle the beam encoder reading only crosses zero once in each direction. However, the motor encoder measurement, particularly when damping is added, crosses zero multiple times. The measurement error caused by the vibrations can cause unstable behaviour by causing the controller to think the end effector has left the haptic wall whilst it is still in it and vice versa. Therefore, it seems that the stiffness of the motor shaft is a factor in whether the end effector of the haptic device can to come to rest near or at a stiff virtual wall.

The gyro damping was seen to be more effective whilst using the motor encoder and only marginally better whilst using the beam encoder. Figure 5 shows the gyro damps the limit cycles of the haptic device more effectively but does not remove the vibration present in the motor at all. When switching to the motor encoder, the vibrations are nearly completely removed and the encoder damping performs far more comparably to the gyro. Figure 8 show this where the amplitude of angular deflection in the shaft is similar in both cases. Since the gyro is more effective at reducing the limit cycle amplitude due to smaller delay and better effective resolution it can be said to be more effective at least when operating below saturation.

\section{Conclusion}

The results show that modelling haptic devices as rigid bodies may not be appropriate in most cases. Even in such a robust haptic device as seen in this experiment, the effect of torsional deflection of the motor shaft result in perceptible disturbances due to vibration which are even audible at high gains. More analysis of haptic devices is needed where key components that exhibit elastic behaviour are modelled as stiff bodies. In this example the vibrations occurred in the motor shaft but in other examples such as a Phantom haptic device, the cable drives are likely to exhibit these kinds of vibrations. In cases such as these, it may be possible to improve stability and reduce unwanted vibration simply by better placement of encoders.

The angular velocity measurement from the gyro sensor resulted in more effective damping at lower velocities relative to the differentiated encoder signal. This performance is only achieved if the bandwidth of the gyro is high enough (> $1000 \mathrm{~Hz}$ ) to accurately measure vibrations at the natural frequency of the system. The gyro rate has several advantages such as higher effective resolution, no delay and does not require filtering. The encoder has limited resolution for measuring angular velocity which is hampered by the high sampling rate, it always incurs a delay from discrete differentiation and is relatively noisy due to the quantisation error and requires filtering adding further control delay. At higher velocities the encoder signal is less noisy and the gyro will begin to saturate and at this point the encoder velocity estimate is preferable.

An alternative approach to haptic device control is to include better models of the elastic properties of the mechanism and use these to provide a better 
estimate of end point position. Of course these models would need to adapt to the passive properties of the human.

\section{References}

1. Hannaford, B., Ryu, J.H.: Time-domain passivity control of haptic interfaces. IEEE Transactions on Robotics and Automation 18(1), 1-10 (2002)

2. Colgate, J., Schenkel, G.: Passivity of a class of sampled-data systems: application to haptic interfaces. In: Proceedings of 1994 American Control Conference - ACC '94. vol. 3, pp. 3236-3240. IEEE (1994), http://ieeexplore.iee.org/document/735172/

3. Wyatt, J.L., Chua, L.O., Gannett, J.W., Goknar, I.C., Green, D.N.: Energy Concepts in the State-Space Theory of Nonlinear n-Ports: Part 11 Losslessness. IEEE Transactions on Circuits and Systems 29(7), 417-430 (1982)

4. Tanaka, H., Ohnishi, K., Nishi, H., Kawai, T., Morikawa, Y., Ozawa, S., Furukawa, T.: Implementation of bilateral control system based on acceleration control using FPGA for multi DOF haptic endoscopic surgery robot. IEEE Transactions on Industrial Electronics 56(3), 618-627 (2009), http://dx.doi.org/10.1109/TIE.2008.2005710

5. Adams, R.J., Moreyra, M.R., Hannaford, B.: Stability and Performance of Haptic Displays : Theory and Experiments. Proceedings ASME International Mechanical Engineering Congress and Exhibition pp. 227-234 (1998)

6. Ryu, J.H., Kim, Y.S., Hannaford, B.: Sampled-and Continuous-Time Passivity and Stability of Virtual Environments. IEEE Transactions on Robotics 20(4), 772-776 (2004)

7. Ryu, J.H., Kwon, D.S., Hannaford, B.: Control of a Flexible Manipulator With Noncollocated Feedback: Time-Domain Passivity Approach. IEEE Transactions on Robotics 20(4), 776-780 (2004)

8. Colgate, J., Grafing, P., Stanley, M., Schenkel, G.: Implementation of stiff virtual walls in force-reflecting interfaces. Proceedings of IEEE Virtual Reality Annual International Symposium pp. 202-208 (1993)

9. Okamura, A.M., Richard, C., Cutkosky, M.R.: Feeling is Believing: Using a ForceFeedback Joystick to Teach Dynamic Systems. Journal of Engineering Education 91(3), 345-349 (jul 2002), http://doi.wiley.com/10.1002/j.2168-9830.2002.tb00713.x 